\title{
The nutraceutical flavonoid luteolin inhibits ADAMTS-4 and ADAMTS-5 aggrecanase activities
}

\author{
Angela Moncada-Pazos • Alvaro J. Obaya • \\ Cristina G. Viloria • Carlos López-Otín • Santiago Cal
}

Received: 16 December 2010 /Revised: 17 February 2011 / Accepted: 17 February 2011 /Published online: 2 March 2011

(C) Springer-Verlag 2011

\begin{abstract}
A disintegrin and metalloprotease with thrombospondin domains (ADAMTS)-4 (aggrecanase-1) and ADAMTS-5 (aggrecanase-2) are metalloproteases involved in articular cartilage degradation and represent potential therapeutic targets in arthritis treatment. We explore herein the ability of different natural compounds to specifically block the destructive action of these enzymes. Following a preliminary screening using carboxymethylated transferrin as substrate, we focused our interest on luteolin due to its inhibitory effect on ADAMTS-4 and ADAMTS-5 activities using aggrecan and fluorogenic peptides as substrates. However, matrix metalloproteinases (MMPs) activities on these substrates result less affected by this flavonoid. Moreover, incubation of mouse chondrogenic ATDC5 cells in the presence of luteolin clearly decreases the release of aggrecan fragments mediated by aggrecanases under the same conditions in which aggrecanolysis mediated by MMPs is detected. Additionally, glycosaminoglycan levels in culture medium of murine cartilage explants stimulated with interleukin-1-alpha plus retinoic acid are reduced by the presence of the flavonoid. This inhibition takes place
\end{abstract}

A. Moncada-Pazos · C. G. Viloria · C. López-Otín · S. Cal Departamento de Bioquímica y Biología Molecular, Instituto Universitario de Oncología, Universidad de Oviedo, 33006 Oviedo, Spain

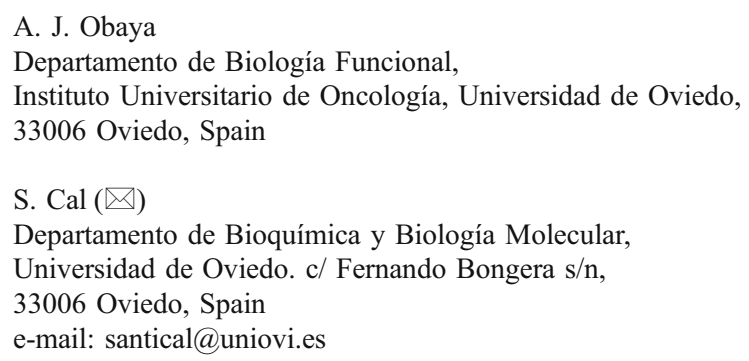

through blockade of ADAMTS-mediated aggrecanolysis, while MMPs activity is not or poorly affected. These results suggest that luteolin could be employed as a prototypic modifying disease-agent to create new chondroprotective compounds aimed to specifically block the unwanted aggrecanase activities in arthritic diseases.

Keywords Arthritis · Biochemistry · Inflammation . Extracellular matrix

\section{Introduction}

Proteolytic degradation of articular cartilage is a key pathological feature of arthropathies such as osteoarthritis (OA) and rheumatoid arthritis (RA) [1]. Particularly, degradation of aggrecan and type II collagen represents a hallmark of arthritis pathology [2]. Aggrecan is a proteoglycan with a core protein of high molecular mass $(\sim 250 \mathrm{kDa})$, consisting of two globular domains at the $\mathrm{NH}_{2}$ terminus and a single globular domain at the $\mathrm{COOH}$ terminus, separated by a large domain heavily modified with glycosaminoglycans (GAG) [3]. The region linking the two $\mathrm{NH}_{2}$ terminus globular domains is the interglobular (IGD) domain, which is highly sensitive to proteolysis. Thus, nine different cleavage sites within this domain have been characterized following aggrecan incubation with purified proteases. Nevertheless, only two major cleavages appear to occur in human tissues [3]. One site is between VDIPEN $^{341}$ and ${ }^{342} \mathrm{FFGVG}$ and this cleavage is attributed to the action of different members of the matrix metalloproteinase (MMP) family. The other site is between NITEGE ${ }^{373}$ and ${ }^{374}$ ARGSVI and is cleaved by ADAMTSs (a disintegrin and metalloprotease with thrombospondin domains) [1]. The functional consequences of aggrecan 
cleavage are distinct depending on the site undergoing proteolytic breakdown. Thus, while the involvement of MMPs in arthritis through type II collagen cleavage is well established [4], their role in aggrecanolysis remains controversial. Some studies have suggested that MMPs, through the $\mathrm{N}^{341}{ }^{342} \mathrm{~F}$ cleavage of aggrecan [4], are involved in the normal cartilage turnover whereas ADAMTS cleavage at $\mathrm{E}^{373}{ }^{374} \mathrm{~A}$ plays a major role in pathological loss of aggrecan from cartilage.

Functional studies support the main role of two members of the ADAMTS family, ADAMTS-4 (aggrecanase-1) and ADAMTS-5 (aggrecanase-2), in pathological aggrecanolysis [5]. Indeed, mice lacking MT1-MMP [6] or MMP-9 [7] show increased cartilage degradation, whereas Adamts5null mice are protected from cartilage degradation [8]. These and other studies have highlighted that therapeutic blockade of aggrecanases could be an option for arthritis treatment [5]. In this regard, versatile health benefits of nutraceutical flavonoids indicate that these compounds might be employed as anti-inflammatory compounds [9]. Moreover, several flavonoids have emerged as potential anti-arthritis drugs due to their metalloprotease inhibitory activities. For instance, a series of green tea catechin gallate ester has been found effective against different members of the MMP and ADAMTS families [10]. Also, nobiletin, a flavonoid isolated from citrus peels, effectively interferes with Adamts 4 and Adamts5 gene expression and hence, aggrecan degradation mediated by these enzymes is hampered by treatment with this flavonoid [11].

The present study has examined the inhibition of aggrecanases by luteolin, a flavonoid widely distributed in the plant kingdom, especially in celery and green pepper [12]. Healthy benefits of luteolin have been evaluated indicating that this flavonoid might protect against cancer, microbial infections, and some chronic diseases [12]. Different mechanisms have been associated with the antiinflammatory properties of luteolin, including inhibition of lipopolysaccharide-induced interleukin (IL)-6 production [13] or NF-kappa B activation [14]. In this work, we report that luteolin can also inhibit aggrecan degradation mediated by ADAMTS-4 and ADAMTS-5. Moreover, we have employed different biochemical approaches, cell-based assays, and murine cartilage explants to determine that luteolin is a better inhibitor of aggrecanases than of MMPs.

\section{Materials and methods}

\section{Materials}

Dulbecco's Eagle's medium (DMEM/F12), fetal bovine serum (FBS), antibiotics, and glutamine were from Life Technologies, Inc., (Paisley, UK). Human apo-transferrin and sodium selenite, chondroitinase $\mathrm{ABC}$ from Proteus vulgaris, keratanase from Pseudomonas sp., aggrecan from bovine articular cartilage, retinoic acid, dimethylmethylene blue, and human transferrin were from Sigma-Aldrich (St. Louis, MO, USA). Transferrin was carboxymethylated using nonradioactive iodoacetic acid [15]. BC-3 antibody was from Abcam (Cambridge, United Kingdom), and BC-14 was from MD Biosciences (Zürich, Switzerland). Curcumin, resveratrol, z-guggulsterone, piceatannol, luteolin, genistein, GM6001 (Ilomastat), dissolved in dimethylsulfoxide (DMSO) following manufacturer's indications; and recombinant MMP-7 were from Calbiochem (Darmstadt, Germany). Recombinant MMP-2, MMP-13, ADAMTS-4, ADAMTS-5, IL-1 $\beta$, IL- $1 \alpha$, and the fluorogenic peptide Mca-K-P-L-G-LDpa-A-R-NH $\mathrm{N}_{2}$ (substrate IX) were from R\&D Systems (Minneapolis, MN, USA). SensoLyte ${ }^{\circledR} 520$ Aggrecanase-1 Assay Kit was from AnaSpec (Fremont, CA, USA). Fluorogenic peptide Dabcyl-K-E-L-A-E-L-R-E-S-T-S-Glu (Edans) (TS5-peptide, sequence taken from peptide FasL1, patent US7,312,045B2, www.freepatentsonline.com) was from JPT Peptide Technologies (Berlin, Germany). ATDC5 cells were a generous gift from Dr. Oreste Gualillo (Complejo Hospitalario Universitario de Santiago de Compostela, Spain).

\section{Enzymatic assays}

Proteolytic assay on carboxymethylated transferrin (Cm-Tf) $(60 \mu \mathrm{M})$ was carried out for $16 \mathrm{~h}$ at $37^{\circ} \mathrm{C}$ in $15 \mu \mathrm{L}$ aggrecanase reaction buffer (ARB; $50 \mathrm{mM}$ Tris- $\mathrm{HCl}$ at pH 8.5 containing $150 \mathrm{mM} \mathrm{NaCl}, 10 \mathrm{mM} \mathrm{CaCl}_{2}, 0.02 \%$ $\mathrm{NaN}_{3}$, and $0.05 \%$ Brij 35). Metalloproteases were employed at $50 \mathrm{nM}$, with the exception of MMP-7, which was employed at $40 \mathrm{nM}$. Reactions were stopped by addition of reducing SDS-PAGE sample buffer containing $20 \mathrm{mM}$ EDTA. Digestion products were analyzed by SDSPAGE gels and stained using Coomassie Brilliant Blue R-250. For aggrecanase assays, recombinant aggrecan $(20 \mu \mathrm{g})$ was incubated with the indicated enzymes in $100 \mu \mathrm{L}$ of ARB. Reactions were stopped by adding an equal volume of double strength glycosaminoglycan buffer (200 mM sodium acetate, $50 \mathrm{mM}$ Tris- $\mathrm{HCl} \mathrm{pH} 6.8$, and $100 \mathrm{mM}$ EDTA). Samples were deglycosylated with 0.01 units of chondroitinase $/ 10 \mu \mathrm{g}$ of aggrecan, and 0.01 units of keratanase $/ 10 \mu \mathrm{g}$ aggrecan for $16 \mathrm{~h}$ at $37^{\circ} \mathrm{C}$, and then precipitated with acetone and centrifuged at $3,000 \times \mathrm{g}$ for $15 \mathrm{~min}$. Pellets were dried and resuspended in $20 \mu \mathrm{L}$ of reducing SDS-PAGE sample buffer. Samples were monitored by Western-blot analysis in polyvinylidene fluoride (PVDF) membranes with $\mathrm{BC}-3$ or $\mathrm{BC}-14$ antibodies [16]. Blots were scanned and the band intensity quantified using ImageJ program. Kinetic analysis using fluorogenic peptide were made in a LS55 PerkinElmer Life 
Science spectrofluorometer $\left(\lambda_{\mathrm{ex}}=320 \mathrm{~nm}\right.$ and $\lambda_{\mathrm{em}}=405 \mathrm{~nm}$ for the fluorogenic peptide IX, and $\lambda_{\mathrm{ex}}=340 \mathrm{~nm}$ and $\lambda_{\mathrm{em}}=$ $500 \mathrm{~nm}$ for TS5-peptide). Routine assays were performed using a substrate concentration of $10 \mu \mathrm{M}$ for peptide IX and $50 \mu \mathrm{M}$ for TS5-peptide. Metalloproteases were assayed at $10 \mathrm{nM}$ and the enzymatic activity detected as increase in fluorescence at $37^{\circ} \mathrm{C}$ for different times. ADAMTS-4 activity was assayed using the SensoLyte ${ }^{\circledR} 520$ Aggrecanase-1 Assay Kit according to manufacturer's instructions. DMSO concentration in the enzymes assays was $<1 \%$. When indicated, preincubation with the specified concentrations of the different inhibitors was performed for $30 \mathrm{~min}$ at $37^{\circ} \mathrm{C}$. $\mathrm{IC}_{50}$ values were calculated from plots of activity versus inhibitor concentration in a semilogarithmic representation.

\section{Chondrocyte cultures}

ATDC5 cells were routinely maintained in DMEM/F12 medium supplemented with 5\% FBS, antibiotics, glutamine, apo-transferrin, and sodium selenite [17]. To evaluate aggrecanolysis, ATDC5 cells were cultured for $48 \mathrm{~h}$ in serum-free DMEM/F12 containing $100 \mu \mathrm{g} / \mathrm{mL}$ aggrecan. When indicated, IL-1 $\beta$ (10 ng/mL) and/or luteolin $(10 \mu \mathrm{M})$ were added to the cultures. Media quenched with EDTA prior to incubation with the substrate served as assay blanks [18]. To evaluate aggrecan degradation products in chondrocyte cultures, $1 \mathrm{~mL}$ of conditioned medium was precipitated with five volumes of acetone and deglycosylation was carried out as described above. For comparative studies, all PVDF membranes were processed simultaneously and Western analysis was performed under identical conditions. Vehicle only (DMSO) was added to control cells not treated with luteolin, to ensure that the observed effects are ascribable to luteolin.

Analysis of glycosaminoglycans release using cartilage explants

Murine femoral head cartilages from 4-week-old mice were harvested and placed into a 48-well culture plate. Explants were maintained in a humidified incubator as previously described [19]. After 2 days, explants were placed in serum-free medium and stimulated or not with IL- $1 \alpha$ $(10 \mathrm{ng} / \mathrm{mL})$ and a retinoic acid $(10 \mu \mathrm{M})$, in the presence or absence of $100 \mu \mathrm{M}$ luteolin. Glycosaminoglycan content in the media was measured using the dimethylmethylene blue assay [20]. Results are expressed as the percentage of proteoglycan release relative to the total proteoglycan in the media and digested cartilage. Mouse care and handling were conducted according to the Institutional Animal Care and Use guidelines for animal research.
RNA extraction and RT-qPCR

Total RNA was isolated by guanidium thiocyanatephenol-chloroform extraction and quantified in a NanoDrop. Reverse transcription reactions were carried out with $2.5 \mu \mathrm{g}$ of RNA, using the Thermoscript RT-PCR system with random hexamers and following manufacturer's instructions. Then $4.5 \mu \mathrm{L}$ of a 1:3 dilution of cDNA were employed for qPCR using Taqman hydrolysis probes: Mm00556068_m1 (Adamts4, NM_172845.2), Mm00478620_m1 (Adamts5, NM_011782.2), Mm00439506_m1 (Mmp2, NM_008610.2), Mm01168419 (Mmp 7, NM_010810.4), Mm00439491_m1 (Mmp13, NM_008607.1), and 4352339E (GAPDH) as a reference gene to normalize. TaqMan Master Mix and the 7300 Real-Time PCR System (Applied Biosystems) were employed. PCR conditions were as follows: $50^{\circ} \mathrm{C}$ for $2 \mathrm{~min}$ and $95^{\circ} \mathrm{C}$ for $10 \mathrm{~min}$, followed by 40 cycles of $95^{\circ} \mathrm{C}$ for $15 \mathrm{~s}$ and $60^{\circ} \mathrm{C}$ for $1 \mathrm{~min}$. Relative expression levels were calculated with the $\Delta \Delta C_{\mathrm{q}}$ method, using the BioRad Gene Expression macro for Excel (Microsoft Corporation). Values were calculated referring all expression to the lowest one that was given the value "1." Value " 0 " was given when expression was not detected.

\section{Statistical analysis}

All data are presented as mean \pm SEM. The analysis of released GAGs was done using Student's $T$ test (Prism 5 GraphPad software). $P$ value $<0.05$ was considered significant.

\section{Results}

Inhibitory activity of natural agents against aggrecanases

Curcumin, resveratrol, z-guggulsterone, piceatannol, luteolin, and genistein were selected to examine their relevance as possible aggrecanase inhibitors [21]. These products were initially tested at $5 \mu \mathrm{M}$ on ADAMTS-4 proteolytic activity using $\mathrm{Cm}-\mathrm{Tf}$ as substrate. Ilomastat was included as a positive control for inhibition. Among all these agents, luteolin showed the highest capacity to block ADAMTS-4 activity as a clear reduction of $\mathrm{Cm}$ - $\mathrm{Tf}$ degradation can be observed with respect to the reaction without the flavonoid (Fig. 1a). Similar results were obtained when using ADAMTS-5 (not shown). Then, we analyzed the capacity of luteolin to inhibit aggrecanases in comparison to MMPs. As can be observed in Fig. 1b, the presence of this flavonoid hampers the proteolytic activity of both ADAMTS-4 and ADAMTS-5 on Cm-Tf in the micromolar range. Pixel density analysis allowed calculating an $\mathrm{IC}_{50}$ 
of $\sim 10 \mu \mathrm{M}$, and degradation of this substrate was totally abolished at $50 \mu \mathrm{M}$ luteolin (Fig. 1b). However, MMP-2, MMP-7, and MMP-13 remain active at concentrations up to $100 \mu \mathrm{M}$ luteolin (Fig. 1b). These results indicate that luteolin greatly influences aggrecanase activities, but MMPs activities result less affected in the micromolar concentration range employed. In consequence, luteolin
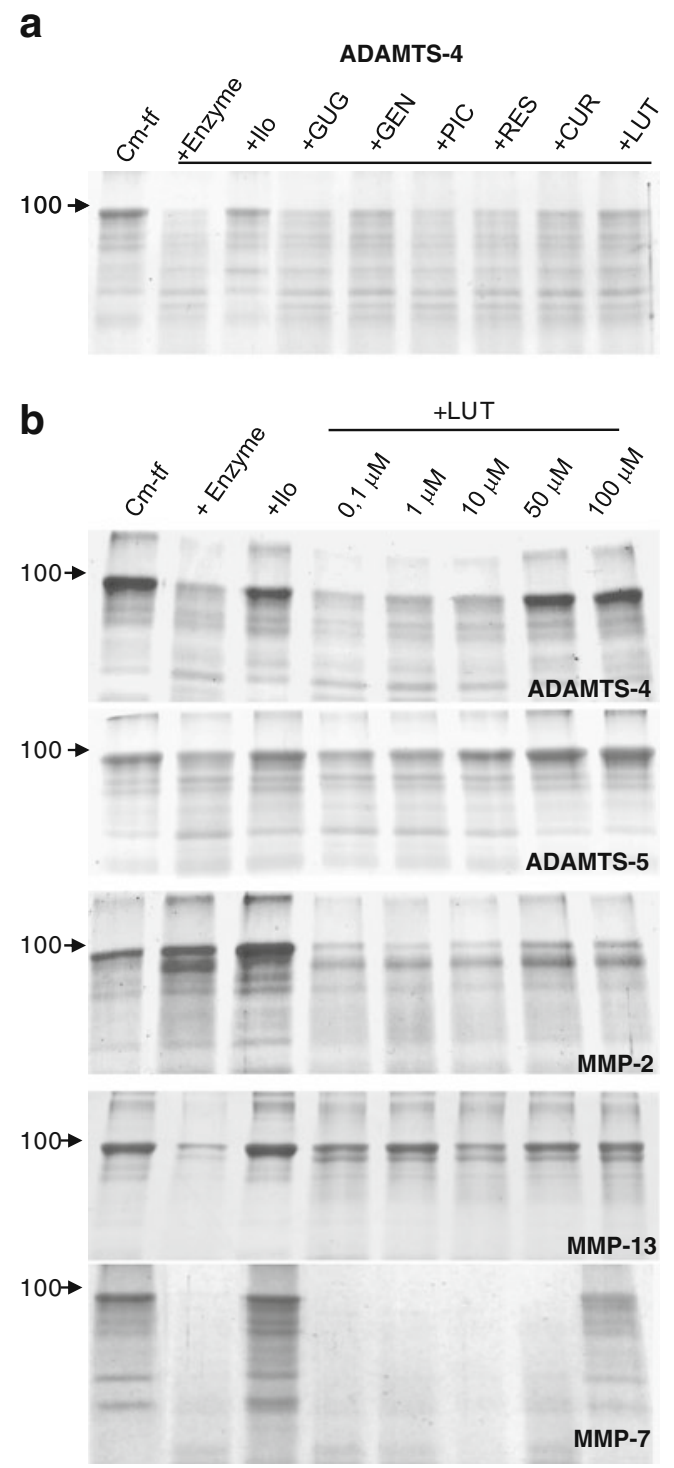

Fig. 1 Luteolin inhibits $\mathrm{Cm}-\mathrm{Tf}$ cleavage by aggrecanases. a SDS-PAGE showing the potential ability of different natural products to block ADAMTS-4 enzymatic activity on Cm-Tf. Lane $1 \mathrm{Cm}$-Tf alone, Enzyme indicates Cm-Tf degradation by ADAMTS-4 without preincubation with any agent. + Ilo indicates that the enzyme was preincubated with Ilomastat. The remaining lanes show the results following preincubation with z-guggulsterone $(+G U G)$, genistein $(+G E N)$, piceatannol $(+P I C)$, resveratrol $(+R E S)$, curcumin $(+C U R)$, and luteolin $(+L U T)$. b Effect of luteolin on aggrecanase and MMP activities. ADAMTS-4, ADAMTS-5, MMP-2, MMP-13, and MMP-7 were preincubated with luteolin $(+L U T)$ at the indicated concentrations. Please note that the metalloproteases were employed at $50 \mathrm{nM}$, with the exception of MMP-7, which was employed at $40 \mathrm{nM}$ could be a potential inhibitor of the ADAMTS-mediated proteolysis.

Luteolin inhibits ADAMTS-mediated aggrecan cleavage

Then, we wanted to examine whether luteolin could also inhibit ADAMTS-4 and ADAMTS-5 activity on aggrecan. To this end, we employed bovine aggrecan as substrate and aggrecanolytic activities were detected using BC-3 antibody. This antibody recognizes the newly generated aminoterminal ${ }^{374}$ ARGSVI sequence following digestion of the IGD of aggrecan by different ADAMTSs (Fig. 2a), but does not recognize this sequence when present within a peptide spanning the cleavage site [3]. Aggrecan catabolites following degradation by ADAMTS-4 or ADAMTS-5 were detected as immune reactive bands at $\sim 250 \mathrm{kDa}$ (Fig. 2b). To examine whether luteolin could restrain these degradative activities, aggrecanases were preincubated with the flavonoid at 1,10 , and $50 \mu \mathrm{M}$, respectively. Result in Fig. $2 \mathrm{~b}$ shows that aggrecan degradation products are scarcely detected when luteolin is present at $10 \mu \mathrm{M}$ and essentially absent at $50 \mu \mathrm{M}$, indicating an inhibitory effect of the flavonoid on ADAMTS activities.

To evaluate whether luteolin could affect aggrecan digestion by MMPs, aggrecan was incubated with MMP13 and MMP-7 in the presence or absence of the flavonoid, and degradation products were detected using BC-14. This antibody recognizes the ${ }^{342} \mathrm{FFGVG}$ amino-terminal sequence generated by MMPs degradation of IGD domain of aggrecan (Fig. 2a). In the absence of the flavonoid, BC-14 immunoreactive fragments following MMP-13 digestion were mainly detected at $\sim 150 \mathrm{kDa}$. In the case of MMP-7, major degradation products were detected at $\sim 150$ and $\sim 70 \mathrm{kDa}$ (Fig. 2b). Preincubation of both MMPs with luteolin at the same concentrations assayed for ADAMTSs caused a slight but not significant decrease of MMP activities as BC-14 immunoreactive bands can be detectable at $50 \mu \mathrm{M}$ luteolin.

Peptide substrate assays

Fluorogenic peptide Mca-K-P-L-G-L-Dpa-A-R-NH $\mathrm{N}_{2}$ was employed to assess the inhibitory effect of luteolin on MMP-2, MMP-7, and MMP-13. Data were fit to sigmoid curve to calculate $\mathrm{IC}_{50}$ values. Results indicate that luteolin is a better inhibitor for MMP-13 $\left(\mathrm{IC}_{50}=18.3 \mu \mathrm{M}\right)$ than for MMP-2 and MMP-7 ( $\mathrm{IC}_{50}=40.9$ and $68.2 \mu \mathrm{M}$, respectively; Fig. 3a). The above peptide is not a suitable substrate for ADAMTSs, and hence the SensoLyte ${ }^{\circledR} 520$ Aggrecanase-1 Assay Kit was employed to calculate an $\mathrm{IC}_{50}$ value of $9.8 \mu \mathrm{M}$ for luteolin, highlighting the inhibitory effect of this flavonoid on ADAMTS-4. To evaluate ADAMTS-5 activity in the presence of luteolin, the fluorogenic TS5-peptide was 
Fig. 2 Effect of luteolin on aggrecan digestion by aggrecanases and MMPs. a Aggrecanases (ADAMTS) and MMPs cleavage sites in the aggrecan IGD domain. $G 1, G 2$, and $G 3$ indicate aggrecan globular domains 1,2, and 3, respectively; $K S$, keratan sulphate; $C S$, chondroitin sulphate. The newly generated amino-terminal sequences following aggrecanases or MMPs digestion are indicated. $\mathbf{b}$

Western-blot analysis of aggrecan degradation by ADAMTS-4, ADAMTS-5, MMP-13, and MMP-7 in absence $(+$ Enzyme $)$ or presence of luteolin (+Luteolin) at the concentrations indicated in "Results." The ${ }^{374}$ ARGSVI and

${ }^{342}$ FFGVG immunoreactive fragments were detected by the BC-3 and BC-14 antibodies respectively. $C$ indicates aggrecan incubated in the absence of enzyme and luteolin. + Ilo indicates that the corresponding metalloprotease was preincubated with ilomastat a

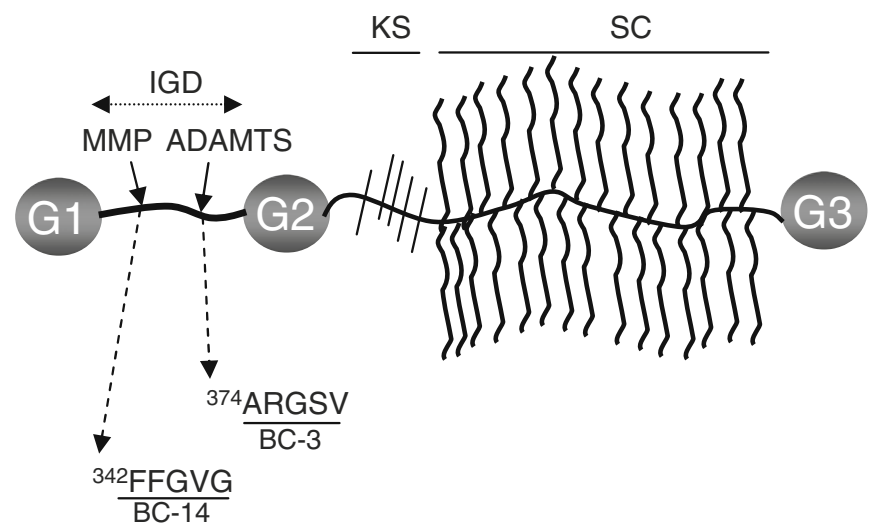

b

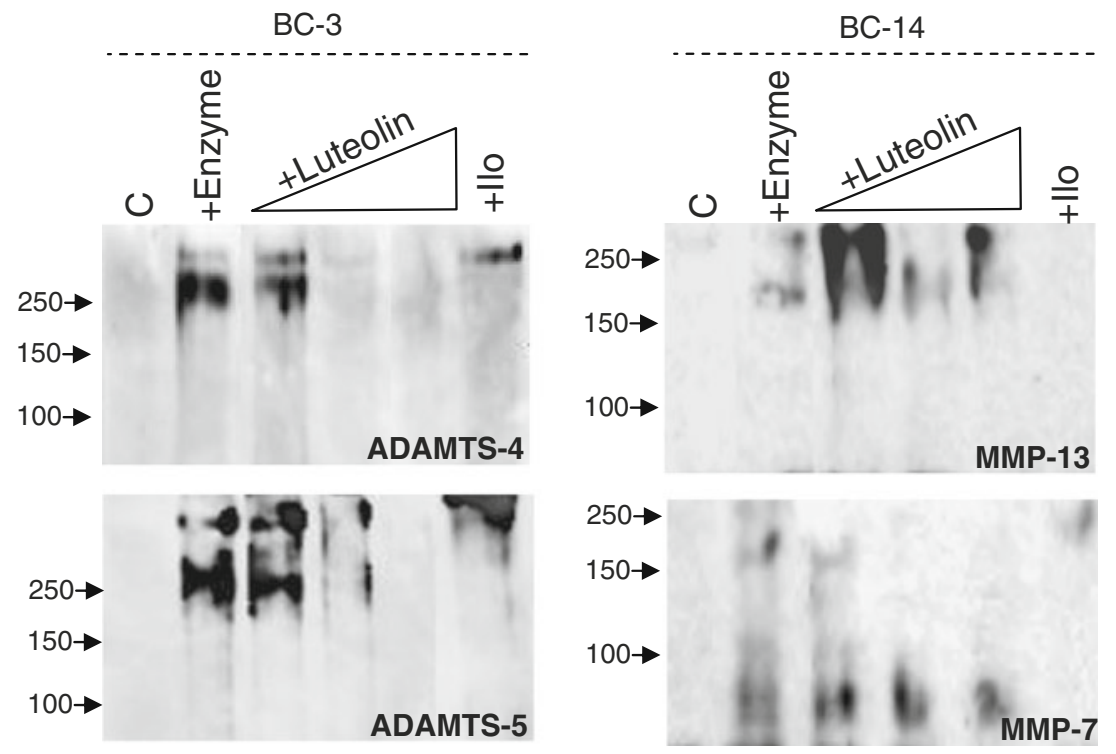

employed and MMP-7 was used for comparative analysis as this MMP hydrolyzes this substrate better than MMP-2 and MMP-13 (not shown). Again, ADAMTS-5 was strongly inhibited by luteolin $\left(\mathrm{IC}_{50}=12.2 \mu \mathrm{M}\right.$, Fig. $3 \mathrm{~b}$ ), whereas the flavonoid did not show a significant inhibitory effect on MMP-7 $\left(\mathrm{IC}_{50}>250 \mu \mathrm{M}\right)$. These substrates were also used to determine that luteolin acts as a noncompetitive inhibitor of ADAMTS-mediated proteolysis (Fig. 3c).

Effects of luteolin on aggrecanolytic activities of ATDC5 cells

ATDC 5 cells were stimulated with IL-1 $\beta$ or left untreated, and then treated or untreated with luteolin. mRNA expression analysis for Adamts4, Adamts5, Mmp2, and Mmp 13 was assessed by qRT-PCR after $48 \mathrm{~h}$ of incubation (Fig. 4a). Luteolin was assayed at $10 \mu \mathrm{M}$, as this concentration is within a range that we had previously examined to discard any effect on ATDC5 cell viability (not shown). This experiment allowed determining expression of Adamts4, Adamts5, and, at lower level, of Mmp13 in the absence of both IL-1 $\beta$ and luteolin. Mmp 7 expression was not detected. The presence of luteolin at $10 \mu \mathrm{M}$ caused a decrease of both Adamts 4 and Adamts 5 expression levels whereas $M m p 2$ expression remained stable, suggesting that this $M M P$ is constitutively expressed. Detectable but low Mmp13 expression was also observed when the flavonoid was present. Similar expression analysis was performed following IL-1 $\beta$ treatment of ATDC5 cells, showing an enhanced expression of Adamts4, Adamts5, and Mmp2, but Mmp13 expression was again undetectable in the absence of luteolin (Fig. 4a). However, combination in the culture of both the flavonoid and the cytokine moderately decreased Adamts4 and Mmp2 expression, whereas Adamts5 expression was markedly reduced respecting the cells grown in the presence of IL-1 $\beta$ alone. Similarly to what happened in the absence of IL-1 $\beta, M m p 13$ expression is detected at a low expression level in the presence of luteolin. 
Fig. 3 Effect of luteolin on the digestion of fluorogenic peptide substrates by aggrecanases and MMPs. a Fluorogenic peptide substrate IX for MMP-2, MMP7, and MMP-13, and the SensoLyte ${ }^{\circledR} 520$ Aggrecanase-1 Assay Kit for ADAMTS-4 were used for monitoring metalloprotease activities in the presence of various concentrations of luteolin as described in "Materials and Methods." b Similar assay using the fluorogenic TS5peptide, a common substrate for MMP-7, and ADAMTS-5.

Dashed lines indicate $50 \%$ ctivity. c Lineweaver-Burk plots for ADAMTS-4 and ADAMTS5 determined in the presence of luteolin at the concentrations indicated in brackets
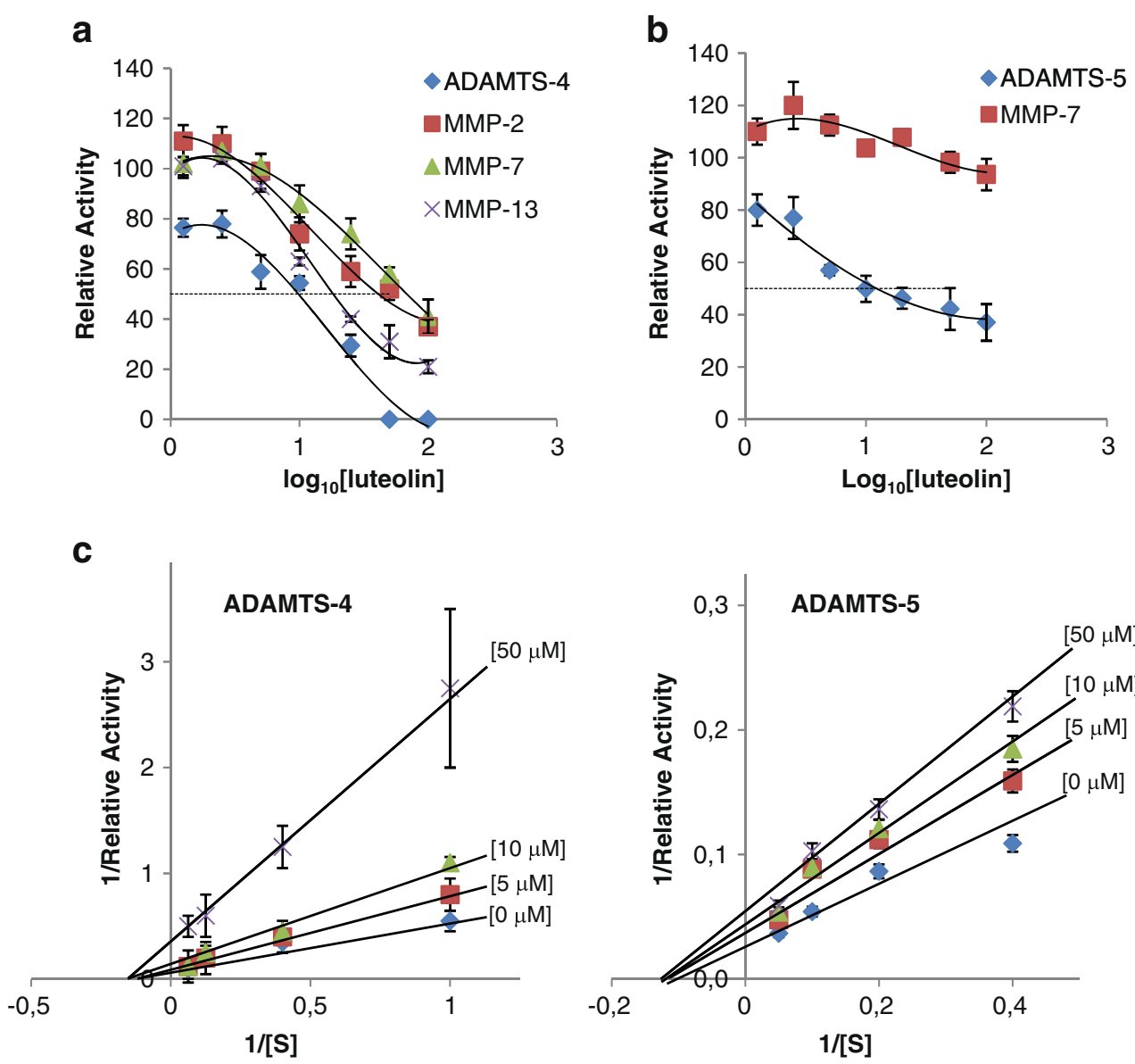

Regarding aggrecanolytic activities, conditioned media were analyzed to detect aggrecan degradation products. Treatment with IL-1 $\beta$ considerably enhanced aggrecanolysis mediated by both ADAMTSs and MMPs, as BC-3 and BC-14 immunoreactive products were increased compared with samples obtained without treatment with the cytokine (Fig. 4b). However, presence of luteolin caused a marked reduction of BC-3 immunoreactivity meanwhile release of high molecular weight aggrecan species was still clearly detected by $\mathrm{BC}-14$. These results indicate that MMPmediated aggrecanolysis is less affected by the presence of the flavonoid. Altogether, these results show that luteolin can act as an aggrecanase inhibitor, and provide novel evidence of how this flavonoid may modulate ADAMTSs gene expression.

\section{Inhibition of glycosaminoglycans release by luteolin}

To evaluate whether luteolin could affect GAG release from cartilage explant, the flavonoid was added to the explant cultures medium of murine femoral head cartilages stimulated with IL- $1 \alpha$ and retinoic acid. Quantification of GAG release from cartilage explants indicates that the presence of luteolin significantly reduces the appearance of these degradation products $(*, p$ value $<0.05$ by Student's $T$ test) in the case of samples stimulated with IL- $1 \alpha$ and retinoic acid (Fig. 5a). Thus, luteolin is indeed diminishing the aggrecanolysis mediated by aggrecanases but not by MMPs (Fig. 5b), thus reinforcing the inhibitory effect of luteolin on these enzymes. For unstimulated samples, the flavonoid slightly increases GAG release (not significant, Fig. 5a). This effect, however, is not statistically significant and cannot be attributable to neither aggrecanases nor MMPs since no products of their degradation were detected (Fig. 5b). Nevertheless, it cannot be ruled out that other GAG-degrading enzymes are also affected by the presence of the flavonoid.

\section{Discussion}

Depletion of aggrecan is a critical event in OA and RA which is mainly caused by uncontrolled proteolytic activities. In this regard, it is well characterized that MMPs cleave aggrecan at the $\mathrm{N}^{341}-{ }^{342} \mathrm{~F}$ bond within the IGD domain, hence these metalloproteases emerged as potential therapeutic targets to block disease progression [5]. However, in 1991, Sandy et al. [22] found that the main cleavage in the aggrecan core protein occurred at $\mathrm{E}^{373}{ }^{374} \mathrm{~A}$ within the IGD domain. This important finding indicated 
a

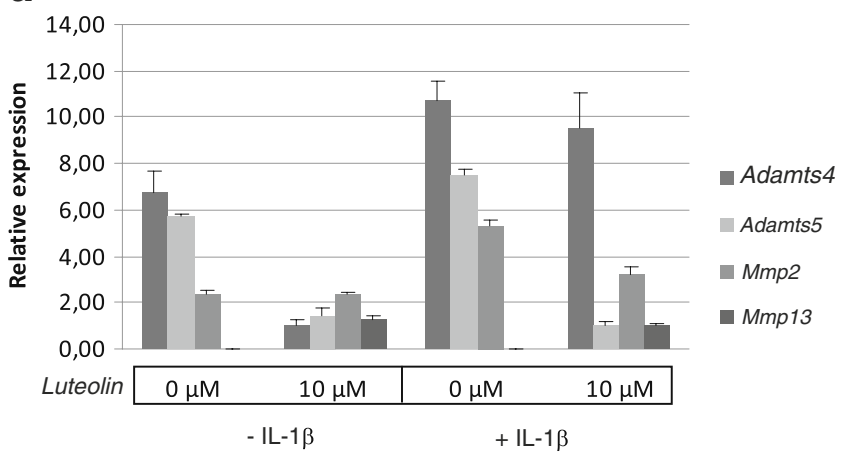

b

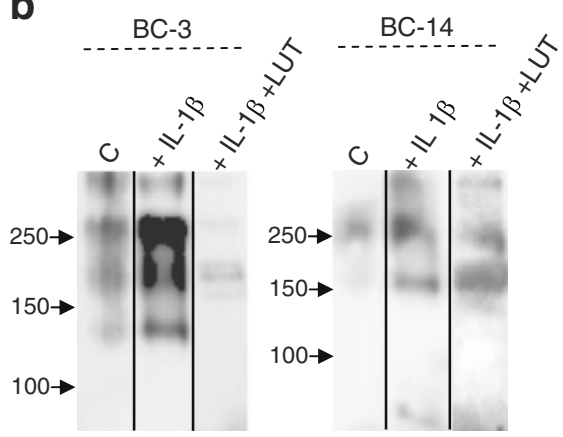

Fig. 4 Inhibitory effect of luteolin on proteoglycan degradation in ATDC5 cells. a Analysis of aggrecanases, Mmp2, and Mmp13 expression in ATDC5 cells in presence or absence of luteolin at the indicated concentration. $+I L-1 \beta$ indicates that cells were treated with this cytokine and $-I L-1 \beta$ that cells were left untreated. No $M m p 7$ expression was found in these assays. b Western-blot analysis of the ${ }^{374}$ ARGSVI and ${ }^{342} \mathrm{FFGVG}$ immunoreactive fragments found in the media of ATDC 5 cells and detected by the BC-3 and BC-14 antibodies. $+I L-1 \beta$ indicates presence of the cytokine, and $+I L-1 \beta+L U T$ indicates presence of both cytokine and luteolin. $C$ indicates absence of both IL-1 $\beta$ and luteolin

that proteolytic enzymes other than MMPs might be involved in pathological aggrecan degradation and therefore this proteolytic activity was referred as aggrecanase. Two ADAMTS metaloproteases, ADAMTS-4 (aggrecanase-1) and ADAMTS-5 (aggrecanase-2), were identified as the main enzymes responsible for the cleavage at this $\mathrm{E}^{373}{ }^{374} \mathrm{~A}$ bond [5]. Functional implication of ADAMTS-5 in arthritis has been further demonstrated by the phenotypic characterization of Adamts5-deficient mouse, which has revealed that this metalloprotease is the main responsible for cartilage degradation in vivo [8].

The identification of ADAMTS-4 and ADAMTS-5 as pathological aggrecanases has increased the interest in the discovery and/or design of specific inhibitors [5]. Synthetic hydroxamic acid-derived compounds such as ilomastat are potent ADAMTSs inhibitors though their zinc-chelating ability. These products were originally designed to block MMPs to avoid the uncontrolled activity of these metalloproteases in tumorigenesis [23]. However, their use in anticancer therapies resulted disappointing since different a

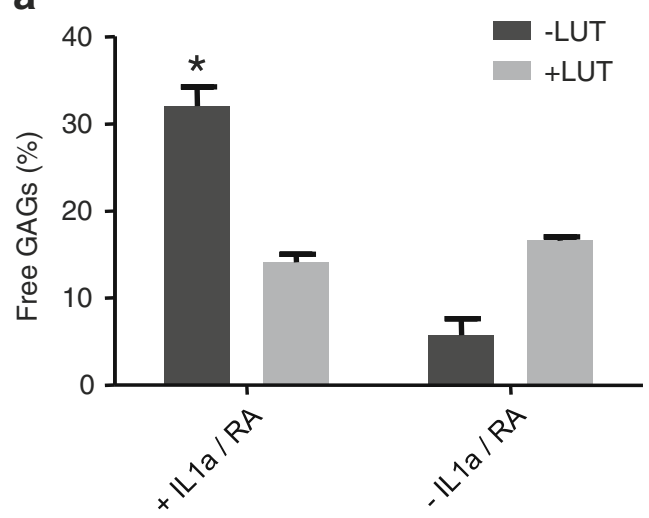

b

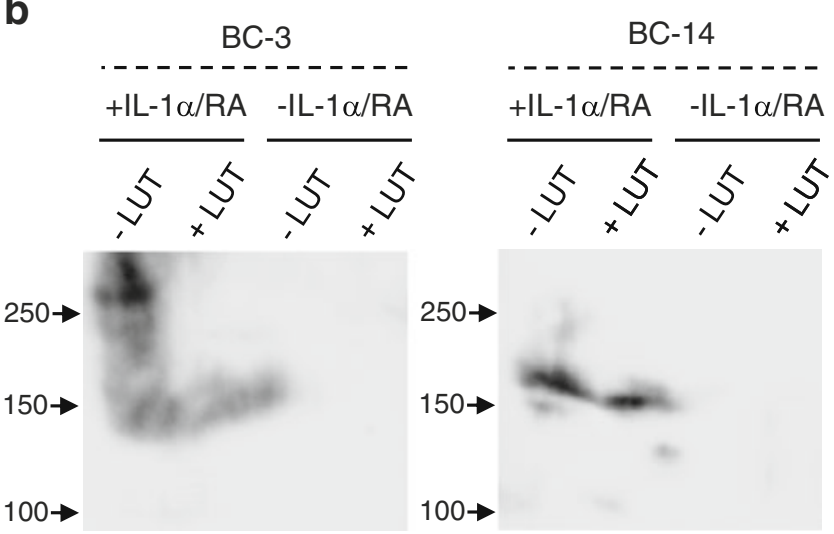

Fig. 5 Inhibition of cartilage glycosaminoglycans release by luteolin. a Quantification of GAGs release was carried out using the dimethylmethylene blue assay. Cartilage explants were stimulated with IL- $1 \alpha$ and retinoic acid ( + IL- $1 \alpha /$ retinoic acid), or were left unstimulated (-IL-1 $\alpha /$ retinoic acid). $+L U T$ indicates presence of luteolin. $\left({ }^{*} p\right.$ value $<0.05$ by Student's $T$ test). b Western-blot analysis of the aggrecan fragments generated by ADAMTS $(B C-3)$ or MMPs $(B C-14)$ activity in cartilage explants stimulated (+IL- $1 \alpha /$ retinoic acid) or not (-IL- $1 \alpha /$ retinoic acid), with $\mathrm{IL}-1 \alpha$ and retinoic acid in presence $(+L U T)$ or absence $(-L U T)$ of luteolin

MMPs show protective roles in tumor progression [24] and because their lack of specificity implies that other tumorprotective metalloproteases are also affected [25-27]. Similarly, the use of these broad spectrum inhibitors would not be suitable in the case of arthritic diseases. Under pathological conditions, a proinflammatory environment would predominate, and ADAMTS-4 and ADAMTS-5 would deteriorate the cartilage by degrading its components. On the other side, MMPs would be necessary for normal tissue remodeling. Hence, the inhibition of ADAMTSs would prevent cartilage degradation, while blocking MMPs would affect normal cartilage turnover. Consequently, new inhibitors aimed to selectively block ADAMTS activities are necessary. In this regard, a possibility would consist in targeting the exosite interactions of these enzymes. In fact, different studies have shown that aggrecan cleavage depends on the numerous 
interactions occurring between the core enzyme outside the active site and the substrate [28]. Thus, calcium pentosan polysulfate (CaPPS) inhibits ADAMTS-4 aggrecanase activity without affecting mRNA expression in chondrocytes treated with IL-1 or retinoic acid [29]. Chondroprotective role of CaPPS could occur through its binding with the carboxy-terminal domain of the protease, avoiding a proper interaction with the substrate. Similarly, glucosamine and manosamine also inhibit aggrecan degradation in chondrocyte cultures and it has been suggested that these compounds prevent mechanical changes occurring to the cartilage following IL-1 treatment [30].

In this work, we have explored whether different nutraceutical compounds could have an inhibitory effect on ADAMTS- 4 and -5 activities as numerous natural products have proven to exert anti-inflammatory effects in vitro and in vivo. For instance, it has been recently shown that licochalcone A, a major phenolic constituent of licorice, decreases levels of proinflammatory cytokines in mouse models [31]. We first performed a screening to identify natural products able to target ADAMTS-4 and ADAMTS-5 activity. These products included piceatannol, a polyphenol with ability to act as an ADAMTS-4 inhibitor [32]. However, our results indicated that luteolin has the highest capacity to block these enzymes among all products assayed, whereas degradation mediated by MMPs was less affected by this flavonoid. In this regard, it has been previously reported that a MMP-2 form including the catalytic domain with the fibronectin-like repeats can be inhibited by luteolin [33]. Nevertheless, in this work, we have employed an entire form of the protein, including all ancillary domains and requiring activation to perform its catalytic activity. Differences in the level of inhibition obtained between these two MMP-2 forms could indicate the relevance of the ancillary domains in the recognition of the substrate and consequently for the catalytic process. Luteolin also possesses anti-inflammatory activity, which is mediated by inhibiting the production of cytokines such as IL-8, IL-15, or TGF- $\beta$ in synovial fibroblasts [34], and also by blocking COX-2, iNOS, LOX [12], and IL-6 [13] activities. However, to our knowledge, it had not been yet examined whether luteolin could also act as an aggrecanase inhibitor. In this work, we have shown that this flavonoid inhibits degradation of recombinant aggrecan and its release by chondrocytic cells in vitro, and prevents, ex vivo, the release of glycosaminoglycans from murine femoral head cartilages treated with IL-1 $\alpha$ and retinoic acid. Additionally, we have demonstrated that its inhibitory function is more effective on ADAMTSs than on MMPs activity. Finally, we have provided evidence that luteolin can modulate Adamts gene expression, an observation which is consistent with previous findings on nobiletin, a polymethoxy flavonoid that shows a high structural similarity with luteolin [11].
In conclusion, luteolin is a novel and potential alternative in anti-arthritic therapies, due to its specificity over aggrecanases and to its natural product condition. Thus, the action of this flavonoid would be mainly restricted to the undesired degradation of aggrecan, and its presence in components of normal diet guarantees the possibility of oral administration and non-toxicity at low doses [12]. Moreover, these properties could be improved through the design of new molecules chemically derived from luteolin, which could represent new therapeutic candidates for arthritic diseases [35].

Acknowledgments The authors thank Sonsoles Alvarez for her excellent technical support. This work was supported by grants from Ministerio de Ciencia e Innovación-Spain, Fundación M. Botín and European Union (FP7-MicroEnviMet); The Instituto Universitario de Oncología is supported by Obra Social Cajastur.

Disclosure The authors declare no conflict of interest related to this study.

\section{References}

1. Nagase H, Kashiwagi M (2003) Aggrecanases and cartilage matrix degradation. Arthritis Res Ther 5:94-103

2. Dean DD, Martel-Pelletier J, Pelletier JP, Howell DS, Woessner JF $\mathrm{Jr}$ (1989) Evidence for metalloproteinase and metalloproteinase inhibitor imbalance in human osteoarthritic cartilage. J Clin Invest 84:678-685

3. Arner EC (2002) Aggrecanase-mediated cartilage degradation. Curr Opin Pharmacol 2:322-329

4. Sandy JD (2006) A contentious issue finds some clarity: on the independent and complementary roles of aggrecanase activity and MMP activity in human joint aggrecanolysis. Osteoarthritis Cartilage 14:95-100

5. Fosang AJ, Little CB (2008) Drug insight: aggrecanases as therapeutic targets for osteoarthritis. Nat Clin Pract Rheumatol 4:420-427

6. Zhou Z, Apte SS, Soininen R, Cao R, Baaklini GY, Rauser RW, Wang J, Cao Y, Tryggvason K (2000) Impaired endochondral ossification and angiogenesis in mice deficient in membrane-type matrix metalloproteinase I. Proc Natl Acad Sci USA 97:40524057

7. Colnot C, Thompson Z, Miclau T, Werb Z, Helms JA (2003) Altered fracture repair in the absence of MMP9. Development 130:4123-4133

8. Stanton H, Rogerson FM, East CJ, Golub SB, Lawlor KE, Meeker CT, Little CB, Last K, Farmer PJ, Campbell IK et al (2005) ADAMTS5 is the major aggrecanase in mouse cartilage in vivo and in vitro. Nature 434:648-652

9. Colic M, Pavelic K (2000) Molecular mechanisms of anticancer activity of natural dietetic products. J Mol Med 78:333-336

10. Vankemmelbeke MN, Jones GC, Fowles C, Ilic MZ, Handley CJ, Day AJ, Knight CG, Mort JS, Buttle DJ (2003) Selective inhibition of ADAMTS-1, -4 and -5 by catechin gallate esters. Eur J Biochem 270:2394-2403

11. Imada $\mathrm{K}$, Lin N, Liu C, Lu A, Chen W, Yano M, Sato T, Ito A (2008) Nobiletin, a citrus polymethoxy flavonoid, suppresses gene expression and production of aggrecanases- 1 and -2 in collageninduced arthritic mice. Biochem Biophys Res Commun 373:181185 
12. Lopez-Lazaro M (2009) Distribution and biological activities of the flavonoid luteolin. Mini Rev Med Chem 9:31-59

13. Jang S, Kelley KW, Johnson RW (2008) Luteolin reduces IL-6 production in microglia by inhibiting JNK phosphorylation and activation of AP-1. Proc Natl Acad Sci USA 105:7534-7539

14. Xagorari A, Papapetropoulos A, Mauromatis A, Economou M, Fotsis T, Roussos C (2001) Luteolin inhibits an endotoxinstimulated phosphorylation cascade and proinflammatory cytokine production in macrophages. J Pharmacol Exp Ther 296:181-187

15. Nagase H (1995) Human stromelysins 1 and 2. Meth Enzymol 248:449-470

16. Gendron C, Kashiwagi M, Lim NH, Enghild JJ, Thogersen IB, Hughes C, Caterson B, Nagase H (2007) Proteolytic activities of human ADAMTS-5: comparative studies with ADAMTS-4. J Biol Chem 282:18294-18306

17. Otero M, Gomez Reino JJ, Gualillo O (2003) Synergistic induction of nitric oxide synthase type II: in vitro effect of leptin and interferon-gamma in human chondrocytes and ATDC5 chondrogenic cells. Arthritis Rheum 48:404-409

18. Arner EC, Pratta MA, Trzaskos JM, Decicco CP, Tortorella MD (1999) Generation and characterization of aggrecanase. A soluble, cartilage-derived aggrecan-degrading activity. J Biol Chem 274:6594-6601

19. Majumdar MK, Askew R, Schelling S, Stedman N, Blanchet T, Hopkins B, Morris EA, Glasson SS (2007) Double-knockout of ADAMTS-4 and ADAMTS-5 in mice results in physiologically normal animals and prevents the progression of osteoarthritis. Arthritis Rheum 56:3670-3674

20. Barbosa I, Garcia S, Barbier-Chassefiere V, Caruelle JP, Martelly I, Papy-Garcia D (2003) Improved and simple micro assay for sulfated glycosaminoglycans quantification in biological extracts and its use in skin and muscle tissue studies. Glycobiology 13:647-653

21. Khanna D, Sethi G, Ahn KS, Pandey MK, Kunnumakkara AB, Sung B, Aggarwal A, Aggarwal BB (2007) Natural products as a gold mine for arthritis treatment. Curr Opin Pharmacol 7:344-351

22. Sandy JD, Neame PJ, Boynton RE, Flannery CR (1991) Catabolism of aggrecan in cartilage explants. Identification of a major cleavage site within the interglobular domain. J Biol Chem 266:8683-8685

23. Overall CM, Lopez-Otin C (2002) Strategies for MMP inhibition in cancer: innovations for the post-trial era. Nat Rev Cancer 2:657-672
24. Lopez-Otin C, Palavalli LH, Samuels Y (2009) Protective roles of matrix metalloproteinases: from mouse models to human cancer. Cell Cycle 8:3657-3662

25. Lopez-Otin C, Matrisian LM (2007) Emerging roles of proteases in tumour suppression. Nat Rev Cancer 7:800-808

26. Moncada-Pazos A, Obaya AJ, Fraga MF, Viloria CG, Capella G, Gausachs M, Esteller M, Lopez-Otin C, Cal S (2009) The ADAMTS12 metalloprotease gene is epigenetically silenced in tumor cells and transcriptionally activated in the stroma during progression of colon cancer. J Cell Sci 122:2906-2913

27. Viloria CG, Obaya AJ, Moncada-Pazos A, Llamazares M, Astudillo A, Capella G, Cal S, Lopez-Otin C (2009) Genetic inactivation of ADAMTS15 metalloprotease in human colorectal cancer. Cancer Res 69:4926-4934

28. Nagase H, Fushimi K (2008) Elucidating the function of non catalytic domains of collagenases and aggrecanases. Connect Tissue Res 49:169-174

29. Troeberg L, Fushimi K, Khokha R, Emonard H, Ghosh P, Nagase $\mathrm{H}$ (2008) Calcium pentosan polysulfate is a multifaceted exosite inhibitor of aggrecanases. FASEB J 22:3515-3524

30. Patwari P, Kurz B, Sandy JD, Grodzinsky AJ (2000) Mannosamine inhibits aggrecanase-mediated changes in the physical properties and biochemical composition of articular cartilage. Arch Biochem Biophys 374:79-85

31. Kim JK, Shin EK, Park JH, Kim YH (2010) Antitumor and antimetastatic effects of licochalcone A in mouse models. J Mol Med 88:829-838

32. Lauer-Fields JL, Spicer TP, Chase PS, Cudic M, Burstein GD, Nagase H, Hodder P, Fields GB (2008) Screening of potential a disintegrin and metalloproteinase with thrombospondin motifs-4 inhibitors using a collagen model fluorescence resonance energy transfer substrate. Anal Biochem 373:43-51

33. Ende C, Gebhardt R (2004) Inhibition of matrix metalloproteinase-2 and -9 activities by selected flavonoids. Planta Med 70:1006-1008

34. Hou Y, Wu J, Huang Q, Guo L (2009) Luteolin inhibits proliferation and affects the function of stimulated rat synovial fibroblasts. Cell Biol Int 33:135-147

35. Cudic M, Burstein GD, Fields GB, Lauer-Fields J (2009) Analysis of flavonoid-based pharmacophores that inhibit aggrecanases (ADAMTS-4 and ADAMTS-5) and matrix metalloproteinases through the use of topologically constrained peptide substrates. Chem Biol Drug Des 74:473-482 